\title{
DIGITAL TECHNOLOGY Does artificial intelligence (AI) constitute an opportunity or a threat to the future of medicine as we know it?
}

\author{
Author: Misha Kabir ${ }^{\mathrm{A}}$
}

KEYWORDS: Teale essay, AI, artificial intelligence

By far the greatest danger of Artificial Intelligence is that people conclude too early that they understand it.

Eliezer Yudkowsky, Machine Intelligence Research Institute ${ }^{1}$

Artifical intelligence (AI) is the future and is already part of our everyday life. Be it voice recognition assistance with Apple's Siri or Amazon's Alexa, or algorithms that filter your spam emails, recommend a film to you on Netflix, screen your bank account transactions for fraud, or get your auto-pilot flight smoothly to your next holiday destination, chances are you have already experienced AI.

Machine 'deep learning' takes place in a multi-layered 'black box' of deep neural networks, where algorithms are not defined by task-specific rules, but are able to evolve and self-learn using pattern recognition and trial and error. Thus, AI can approach problems as a doctor progressing through their training does: by learning rules from data. However, by having the capacity to analyse massive amounts of data, algorithms are able to find correlations that the human mind cannot.

For some, however, the use of AI in medicine remains a troubling concept. Science fiction is littered with examples of AI running amok at the expense of humanity. While the use of AI in medicine does not evoke exactly the same kind of Orwellian concerns as it might in, for example, national defence, there are still important issues such as privacy, data protection, even the straightforward need for simple human contact to consider. Doctors are expected to temper knowledge with compassion and understanding. These are characteristics which some fear may be lost in any AI driven system, where patients may find their rights are, at best, an afterthought in the relentless pursuit of efficiency.

However, the argument that AI advocates, such as Dr Eric Topol has detailed in his review for the UK's NHS workforce, ${ }^{2}$ is that by allowing for efficiency in the workflow and precision in the practice of medicine, AI will in fact increase the time that patients can spend face-to-face with their healthcare professionals, engaging in shared

Author: ${ }^{\text {A }}$ specialist registrar, St Mark's Hospital and Academic Institute, Harrow, UK decision making. AI will unlikely replace doctors, and the opportunity exists for AI to augment their practise in a number of ways.

> Reducing administrative burden and increasing patientdoctor contact: A 2016 time and motion observational study of 57 US doctors found that they spent about 2 hours doing computer work for every hour spent face-to-face with their patient. ${ }^{3}$ Thirty-seven per cent of the time spent with their patients involved interacting with a computer screen. Voice recognition, natural language processing AI and digital scribing could transform the time spent on clerical tasks, such as writing up clinical notes, ordering tests and prescribing medications from minutes to seconds. AI assistance could markedly reduce the burden of administrative jobs such as coding, billing, scheduling of operating rooms and clinic appointments, and staffing, increasing productivity, saving costs and improving the workflow within healthcare systems.

> Enhancing clinician diagnosis: Deep learning AI can process thousands of radiology or pathology images and conduct automated image interpretation at a fraction of the time that radiologists and pathologists can, allowing for quicker diagnoses. An AI-assisted diagnosis could even allow nonspecialists to confidently make decisions in an emergency setting that would normally require specialist input, for example in fracture $\mathrm{X}$-ray interpretation or cerebral aneurysm detection on computed tomography. Machine learning using tumour genomic sequencing has identified biomarkers that have markedly improved lung cancer classification compared with pathologists using traditional histological data. ${ }^{4}$

However, so far the only AI technologies that have undergone rigorous prospective validation peer-reviewed studies in real-world settings, comparing AI performance to healthcare professionals, exist for the diagnosis of diabetic retinopathy, detection of wrist fractures from X-rays in the emergency department, histologic breast cancer metastases from digitised pathological slides, very small colonic polyps and paediatric cataracts. $^{5}$ These all have shown promise by proving that use of diagnostic algorithms either were as good as or exceeded the accuracy of clinicians, or at least enhanced clinician performance by markedly speeding up time to diagnosis.

$>$ Enhancing monitoring of chronic conditions and patient self-management: Common chronic conditions, such as hypertension, depression and asthma, could 
theoretically be managed in the community with virtual coaching. Smartwatches can now detect arrhythmias such as atrial fibrillation and changes in electrocardiogram morphology can accurately detect hyperkalaemia in patients with chronic kidney disease. AI may also allow monitoring for mental health conditions within the community. Facebook posts have been shown to predict subsequent diagnoses of depression and various tools to detect mood changes such as depression are in development using keyboard interaction, voice and facial recognition and interactive chatbots. ${ }^{6}$

> Predicting future events: Algorithms derived from the interpretation of big data from electronic health records have allowed the creation of a number of risk prediction tools that can be used to alter treatment decisions and prevent poorer outcomes. An AI model was able to self-learn the most optimal treatment for patients with sepsis, using a database of treatment decisions on intravenous fluid resuscitation and vasopressor doses and their impact on survival. ${ }^{6}$ An amount of patient data $(n=13,666)$ could be extracted that far exceeds the lifetime experience accrued by human clinicians. In a large external validation cohort independent of the training data ( $n=79,073$ ), mortality was lower when clinicians' treatment decisions matched those recommended by the predictive model.

Although these examples of how AI could augment medicine are impressive, one of the main obstacles to the widespread acceptance of machine learning in clinical practice is a lack of understanding among patients and their doctors about how the machine's predictions are made. Yet we are willing to accept judgements based on the experience, intuition and cognitive biases of human doctors that cannot always be explicitly explained. We are able to forgive human inaccuracies but have far greater expectations of our machines. This discrepancy in performance expectations may be legitimate given that an inaccurate machine algorithm could have catastrophic consequences on a much larger number of patients than one erroneous physician.

Transparent communication from digital scientists regarding the dataset and methodology used to develop the algorithm, and proper regulation must also be mandated by government legislation. The General Data Protection Regulations emphasises the need for 'explainability' as a key priority in machine learning research. A reorientation of priorities for data scientists are required to now optimise intelligibility of the model to the lay patient in addition to accuracy. Healthcare professionals should also be encouraged to become involved in AI development and validation, through fellowship programmes. Training curricula should be adapted to reflect the need for AI technology intelligibility. Encouraging collaboration between data scientists and clinicians will also benefit the clinical utility of the AI that is developed. Data scientists at present tend to build and evaluate their algorithms based on metrics, such as the best area under the receiver operating characteristic curve, rather than more clinically useful metrics such as high sensitivity or positive predictive value.

Deep learning models rely on large amounts of accurate data but we know that many electronic health records are incomplete which may introduce bias. Algorithms derived from one cohort may become inaccurate when applied to the wider population due to other geographic, societal and care setting differences. For example, an algorithm designed to predict probability of death among hospital patients with pneumonia, spuriously classified asthmatics as low risk. ${ }^{7}$ This was because in the derivation cohort setting, all asthmatic pneumonia care was optimised in an intensive care setting, thus making asthmatics appear to have an above average survival. Therefore, a machine algorithm's validity and causal inferences must be proved through proper prospective trials and randomisation, before acceptance into widespread clinical practice.

AI conceivably provides more opportunity than threat to the future of medicine, but if regulated and understood appropriately. Three main potential sources of big data in medicine are electronic health records, genome sequencing and patient-originating data eg biosensor technology within Fitbits. If this data could be pooled and shared with the scientific community, the capacity to better understand disease aetiology and predict risk outcomes could be astounding. However, legitimate concerns regarding data propriety and security must be allayed by adopting new models of health data ownership with rights to the individual patient, use of highly secure data platforms and governmental legislation.

\section{References}

1 Yudkowsky E. Artificial intelligence as a positive and negative factor in global risk. In: Bostrom N, Cirković MM (eds), Global catastrophic risks. New York: Oxford University Press, 2008: 308-45.

2 Topol E. The Topol Review: Preparing the healthcare workforce to deliver the digital future. Health Education England, 2019.

3 Sinsky C, Colligan L, Li L et al. Allocation of physician time in ambulatory practice: a time and motion study in 4 specialties. Ann Intern Med 2016:165:753-60.

4 Capper D, Jones DTW, Sill M et al. DNA methylation-based classification of central nervous system tumours. Nature 2018;555:469-74.

5 Topol EJ. High-performance medicine: the convergence of human and artificial intelligence. Nat Med 2019;25:44-56.

6 Komorowski M, Celi LA, Badawi O, Gordon AC, Faisal AA. The Artificial Intelligence Clinician learns optimal treatment strategies for sepsis in intensive care. Nat Med 2018;24:1716-20.

7 Watson DS, Krutzinna J, Bruce IN. Clinical applications of machine learning algorithms: beyond the black box. BMJ 2019;364:1886.

Address for correspondence: Dr Misha Kabir, St Mark's Hospital, Northwick Park, Watford Road, Harrow, Middlesex HA1 3UJ, UK.

Email: misha.kabir1@nhs.net 